\title{
Efficacy of platelet transfusion for acute intracerebral hemorrhage among patients on antiplatelet therapy
}

\author{
Onyinyechi Eke, MD*; Mercedeh Shanechi, MD*; Michael Gottlieb, MD, RDMS ${ }^{\dagger}$
}

\begin{abstract}
Clinical question
In patients taking antiplatelet therapy, does a platelet transfusion after acute spontaneous primary intracerebral hemorrhage reduce the risk of death or dependence?

Article chosen

Baharoglu MI, Cordonnier C, Al-Shahi Salman R, et al. Platelet transfusion versus standard care after acute stroke due to spontaneous cerebral hemorrhage associated with antiplatelet therapy (PATCH): a randomized, open-label, phase 3 trial. Lancet 2016;387(10038):2605-13.

Study objective

The primary objective of this study was to investigate whether a platelet transfusion with standard care, compared with standard care alone, reduced death or dependence after intracerebral hemorrhage associated with antiplatelet therapy use.
\end{abstract}

Keywords: intracerebral hemorrhage, antiplatelet therapy, platelet transfusion, PATCH

\section{BACKGROUND}

Hemorrhagic stroke accounts for $11 \%-22 \%$ of strokes, half of all stroke deaths, and significant disability in many of the remaining survivors. ${ }^{1,2}$ Spontaneous and nontraumatic intracerebral hemorrhage (ICH) accounts for two-thirds of all hemorrhagic strokes. ${ }^{3}$ To date, multiple studies had suggested that antiplatelet therapy use before ICH reduce platelet activity and may worsen outcomes, particularly by increasing the risk of early ICH volume growth because of platelet dysfunction. ${ }^{4-6}$ In many clinical settings, platelet transfusion has been used therapeutically for acute ICH, but there is a lack of randomized, controlled clinical trials investigating its effectiveness for reducing death or dependence..$^{7-9}$ While a platelet transfusion has a theoretical benefit in patients with ICH taking antiplatelet therapy, it is important to consider any potential adverse events associated with its use. These complications can include volume overload, febrile and anaphylactoid reactions, thrombotic complications, sepsis secondary to bacterial contamination of platelet products, transfusionrelated acute lung injury, transfusion-associated circulatory overload, and severe anaphylactic episodes. ${ }^{10-11}$ This trial was designed to determine the efficacy of a platelet transfusion among patients with acute $\mathrm{ICH}$ who are treated with antiplatelet therapy.

\section{Population studied}

Patients were enrolled in 36 hospitals in the Netherlands, 13 hospitals in the United Kingdom, and 11 hospitals in France. Patients aged 18 years or older with non-traumatic and supratentorial intracerebral hemorrhage confirmed by brain imaging and a Glasgow coma scale score of 8-15 in whom a platelet transfusion could be initiated within six hours of symptom onset (or last seen well) and within 90 minutes of brain imaging were enrolled. Patients were included if they had been administered antiplatelet therapy with a cyclooxygenase (COX) inhibitor (aspirin or carbasalate calcium), adenosine diphosphate (ADP) receptor inhibitor (clopidogrel), or an adenosine reuptake inhibitor (dipyridamole) for at least seven days preceding the hemorrhage and had a pre-intracerebral hemorrhage modified Rankin Scale (mRS) score of 0 (no symptoms) or 1 (no significant disability). Exclusion criteria were blood on brain imaging suggestive of

From the *Department of Emergency Medicine, Cook County (Stroger) Hospital; and the tDepartment of Emergency Medicine, Rush University Medical Center.

Correspondence to: Dr. Onyinyechi Eke, Department of Emergency Medicine, Cook County (Stroger) Hospital, 1900 W. Polk St., 10th Floor, Chicago, IL 60612; Email: oeke@cookcountyhhs.org 
a subdural or epidural hematoma, an underlying aneurysm, or arteriovenous malformation; planned surgical evacuation of an intracerebral hemorrhage within 24 hours of admission; intraventricular blood that was more than sedimentation in the posterior horns of the lateral ventricles; known use of a vitamin $\mathrm{K}$ antagonist or history of coagulopathy; known thrombocytopenia; a lack of mental capacity based on national legal standards before the intracerebral hemorrhage; or if death appeared imminent. They also did not include participants with infratentorial or large intraventricular hematomas because they were more likely to undergo surgical procedures that might confound the effects of a platelet transfusion on the outcome.

\section{SUMMARY OF METHODS}

\section{Study design}

This was a multicentre, randomized, open-label, and parallel group trial. One-hundred-ninety patients were randomly assigned in a 1:1 ratio to receive either standard care or a platelet transfusion plus standard care within 90 minutes of diagnostic brain imaging. Randomization was performed by investigators using a secure, webbased, and computerized randomization system. Participants and local investigators providing interventions were not masked to treatment allocation, but allocation was concealed to outcome assessors and investigators analyzing data. Standard care was assumed to be provided according to contemporary European and national guidelines. The protocol required a platelet transfusion (one platelet concentrate for a COX inhibitor and two platelet concentrates for an ADP receptor inhibitor) to be initiated within six hours of intracerebral hemorrhage symptom onset and within 90 minutes of diagnostic brain imaging. Functional outcomes were scored at three months using the $\mathrm{mRS}$ and were rated by a neurologist or research nurse who was not involved in the participants' medical treatment. The primary outcome was obtained by the trial coordinating centre for each country. Brain imaging was done 24 hours after randomization. Investigators recorded the occurrence of serious adverse events and other safety outcomes.

\section{Outcomes measured}

The primary outcome studied was a shift toward death or difference in functional outcomes rated with $\mathrm{mRS}$ at three months after randomization. The secondary outcomes were survival (mRS 1-5) at three months, poor outcome defined as a mRS of 4-6 and mRS of 3-6, median absolute intracerebral hemorrhage growth (in $\mathrm{mL}$ ) after 24 hours on brain imaging, and safety. Safety outcomes included complications of a platelet transfusion (e.g., transfusion reactions and thrombotic complications), complications of the intracerebral hemorrhage (e.g., enlargement, intraventricular extension, hydrocephalus, edema, or brain herniation), epileptic seizures, and infection (e.g., urinary tract or pneumonia).

\section{SUMMARY OF RESULTS}

Between 2009 and 2015, 41 sites enrolled 190 participants (97 allocated to platelet transfusion, and 93 allocated to standard care). Baseline characteristics were similar between groups, apart from the rate of peripheral arterial disease that would be unlikely to affect the primary outcome, and no patients were lost to follow-up at three months. Four patients assigned to the intervention group did not receive a platelet transfusion, and two patients assigned to the control group were administered a platelet transfusion.

There was a shift toward higher death and dependence at three months among the platelet transfusion group (adjusted common odds ratio 2.05, 95\% confidence interval [CI 1.18-3.56). Secondary analyses demonstrated a higher ratio of patients with a mRS of 4-6 in the platelet transfusion group, as compared with the standard care group (Table 1). There was no significant difference in the ratio of patients with a mRS of 3-6 or overall survival (Table 1). There was also no significant difference in the median intracranial hemorrhage growth at 24 hours between groups. Forty patients (42\%) who were administered a platelet transfusion had an adverse event, as compared with 28 patients (29\%) in the standard care group.

\section{Study conclusion}

Platelet transfusion appears inferior to standard care among patients administered antiplatelet therapy before an intracranial hemorrhage. A platelet transfusion is not recommended for this indication in clinical practice.

\section{COMMENTARY}

This study was the first randomized, controlled trial to assess the effects of a platelet transfusion on acute ICH 


\begin{tabular}{|c|c|c|c|}
\hline Outcome & Platelet transfusion group (\%) & Standard care group (\%) & Odds ratio $(95 \% \mathrm{Cl})$ \\
\hline Survival at 3 months & $66(68 \%)$ & $72(77 \%)$ & $0.62(0.33-1.19)$ \\
\hline $\mathrm{mRS}$ of $4-6$ at 3 months & $70(72 \%)$ & $52(56 \%)$ & $2.04(1.12-3.74)$ \\
\hline $\mathrm{mRS}$ of $3-6$ at 3 months & $86(89 \%)$ & $76(82 \%)$ & $1.75(0.77-3.97)$ \\
\hline Adverse events & $40(42 \%)$ & $28(29 \%)$ & $1.74(0.96-3.17)$ \\
\hline
\end{tabular}

among patients undergoing antiplatelet therapy. The trial demonstrated worse outcomes among patients who received a platelet transfusion. Regarding the primary endpoint of functional outcome using the mRS at three months, the authors showed that patients who underwent a platelet transfusion had a higher likelihood of death and dependence, as compared with patients who received standard care alone. Additionally, patients who received a platelet transfusion had a greater risk of a moderately severe disability or death (i.e., $\mathrm{mRS} \geq 4$ ) and a higher rate of adverse events.

This is an important finding because it runs contrary to prior observational studies, suggests that a platelet transfusion could be beneficial in these patients, and indicates a change from current practice. ${ }^{12,13}$ Strengths of this study include the involvement of multiple centres in three different countries, completion of clinical follow-up for all participants, adherence to the assigned treatment, and masking of the outcome assessors and investigators analyzing data to the treatment allocation.

It is important to consider several limitations with respect to this study. First, the trial was conducted in three European countries; therefore, it may not be generalizable to other countries with differences in patient populations or current practices. Additionally, most participants in the study had taken aspirin; therefore, the results of the study may not be generalizable to people taking any other antiplatelet therapies. Furthermore, patients with subdural hematomas, epidural hematomas, and aneurysmal bleeding, as well as those likely to require surgical intervention, were excluded, so it is unclear whether a platelet transfusion is more beneficial in this select group. The participants and local investigators giving interventions were not masked to the treatment allocation that may have biased the study. However, this is unlikely given the use of objective criteria and blinding of outcome assessors. Finally, while the study was adequately powered for the primary outcomes based on data from prior studies, it might have been underpowered to detect significant differences in death. ${ }^{14}$

\section{CONCLUSION}

For the treatment of an acute ICH in people taking antiplatelet therapy, platelet transfusion led to higher rates of death and dependence at three months, as well as an increase in serious adverse effects, as compared with patients who received standard therapy. As a result, a platelet transfusion should not be routinely administered to patients with ICH who are on antiplatelet therapy.

Competing interests: None declared.

\section{REFERENCES}

1. Feigin VL, Lawes CM, Bennett DA, Barker-Collo SL, Parag V. Worldwide stroke incidence and early case fatality reported in 56 population-based studies: a systematic review. Lancet Neurol 2009;8(4):355-69.

2. Feigin VL, Krishnamurthi RV, Parmar P, et al. Update on the global burden of ischemic and hemorrhagic stroke in 1990-2013: the GBD 2013 Study. Neuroepidemiology 2015; 45(3):161-76.

3. Al-Shahi Salman R, Labovitz DL, Stapf C. Spontaneous intracerebral haemorrhage. BM7 2009;339:b2586.

4. Thompson BB, Béjot Y, Caso V, et al. Prior antiplatelet therapy and outcome following intracerebral hemorrhage: a systematic review. Neurology 2010;75(15):1333-42.

5. Naidech AM, Jovanovic B, Liebling S, et al. Reduced platelet activity is associated with early clot growth and worse 3-month outcome after intracerebral hemorrhage. Stroke 2009;40(7):2398-401.

6. Davis SM, Broderick J, Hennerici M, et al. Hematoma growth is a determinant of mortality and poor outcome after intracerebral hemorrhage. Neurology 2006;66(8): 1175-81.

7. Kumar A, Mhaskar R, Grossman BJ, et al. Platelet transfusion: a systematic review of the clinical evidence. Transfusion 2015;55(5):1116-27.

8. Kaufman RM, Djulbegovic B, Gernsheimer T, et al. Platelet transfusion: a clinical practice guideline from the AABB. Ann Intern Med 2015;162(3):205-13. 
9. British Committee for Standards in Haematology, Blood Transfusion Task Force. Guidelines for the use of platelet transfusions. Br 7 Haematol 2003;122(1): 10-23.

10. Kiefel V. Reactions induced by platelet transfusions. Transfus Med Hemother 2008;35(5):354-8.

11. Blumberg N, Heal JM, Phillips GL. Platelet transfusions: trigger, dose, benefits, and risks. F1000 Med Rep 2010;2:5.

12. Naidech AM, Liebling SM, Rosenberg NF, et al. Early platelet transfusion improves platelet activity and may improve outcomes after intracerebral hemorrhage. Neurocrit Care 2012;16(1):82-7.

13. Ducruet AF, Hickman ZL, Zacharia BE, et al. Impact of platelet transfusion on hematoma expansion in patients receiving antiplatelet agents before intracerebral hemorrhage. Neurol Res 2010;32(7):706-10.

14. Mendelow AD, Gregson BA, Rowan EN, et al. Early surgery versus initial conservative treatment in patients with spontaneous supratentorial lobar intracerebral haematomas (STICH II): a randomised trial. Lancet 2013;382(9890):397-408. 